\title{
Alpha element abundances and gradients in the Milky Way bulge from FLAMES-GIRAFFE spectra of $650 \mathrm{~K}$ giants ${ }^{\star \star \star \star}$
}

\author{
O. A. Gonzalez ${ }^{1}$, M. Rejkuba ${ }^{1}$, M. Zoccali ${ }^{2}$, V. Hill ${ }^{3}$, G. Battaglia ${ }^{1}$, C. Babusiaux ${ }^{4}$, D. Minniti ${ }^{1,2,8}$, B. Barbuy ${ }^{5}$, \\ A. Alves-Brito ${ }^{2}$, A. Renzini ${ }^{6}$, A. Gomez ${ }^{4}$, and S. Ortolani ${ }^{7}$
}

${ }^{1}$ European Southern Observatory, Karl-Schwarzschild-Strasse 2, 85748 Garching, Germany e-mail: [ogonzale,mrejkuba, gbattagl]@eso.org

2 Departamento Astronomía y Astrofísica, Pontificia Universidad Católica de Chile, Av. Vicuña Mackenna 4860, Stgo., Chile e-mail: [mzoccali, Dante] @astro.puc.cl

3 Université de Nice Sophia Antipolis, CNRS, Observatoire de la Côte d'Azur, BP 4229, 06304 Nice Cedex 4, France e-mail: vanessa.hill@obspm.fr

${ }^{4}$ Observatoire de Paris-Meudon, 92195 Meudon Cedex, France e-mail: [Ana.Gomez, carine.babusiaux]@obspm.fr

5 Universidade de São Paulo, IAG, Rua do Matão 1226, Cidade Universitária, São Paulo 05508-900, Brazil e-mail: Barbuy@astro.iag.usp.br

${ }^{6}$ INAF - Osservatorio Astronomico di Padova, Vicolo dell'Osservatorio 2, 35122 Padova, Italy e-mail: alvio.renzini@oapd.inaf.it

7 Università di Padova, Dipartimento di Astronomia, Vicolo dell'Osservatorio 5, 35122 Padova, Italy e-mail: sergio.ortolani@unipd.it

8 Specola Vaticana, V00120 Citta del Vaticano, Italy

Received 20 January 2011 / Accepted 28 March 2011

\section{ABSTRACT}

\begin{abstract}
Aims. We present the analysis of the $[\alpha / \mathrm{Fe}]$ abundance ratios for a large number of stars at several locations in the Milky Way bulge with the aim of constraining its formation scenario.

Methods. We obtained FLAMES-GIRAFFE spectra $(R=22500)$ at the ESO Very Large Telescope for 650 bulge red giant branch (RGB) stars and performed spectral synthesis to measure $\mathrm{Mg}, \mathrm{Ca}$, Ti, and $\mathrm{Si}$ abundances. This sample is composed of 474 giant stars observed in 3 fields along the minor axis of the Galactic bulge and at latitudes $b=-4^{\circ}, b=-6^{\circ}, b=-12^{\circ}$. Another 176 stars belong to a field containing the globular cluster NGC 6553, located at $b=-3^{\circ}$ and $5^{\circ}$ away from the other three fields along the major axis. Stellar parameters and metallicities for these stars were presented in Zoccali et al. (2008, A\&A, 486, 177). We have also re-derived stellar parameters and abundances for the sample of thick and thin disk red giants analyzed in Alves-Brito et al. (2010, A\&A, 513, A35). Therefore using a homogeneous abundance database for the bulge, thick and thin disk, we have performed a differential analysis minimizing systematic errors, to compare the formation scenarios of these Galactic components.

Results. Our results confirm, with large number statistics, the chemical similarity between the Galactic bulge and thick disk, which are both enhanced in alpha elements when compared to the thin disk. In the same context, we analyze $[\alpha / \mathrm{Fe}]$ vs. $[\mathrm{Fe} / \mathrm{H}]$ trends across different bulge regions. The most metal rich stars, showing low $[\alpha / \mathrm{Fe}]$ ratios at $b=-4^{\circ}$ disappear at higher Galactic latitudes in agreement with the observed metallicity gradient in the bulge. Metal-poor stars $([\mathrm{Fe} / \mathrm{H}]<-0.2)$ show a remarkable homogeneity at different bulge locations.

Conclusions. We have obtained further constrains for the formation scenario of the Galactic bulge. A metal-poor component chemically indistinguishable from the thick disk hints for a fast and early formation for both the bulge and the thick disk. Such a component shows no variation, neither in abundances nor kinematics, among different bulge regions. A metal-rich component showing low $[\alpha / \mathrm{Fe}]$ similar to those of the thin disk disappears at larger latitudes. This allows us to trace a component formed through fast early mergers (classical bulge) and a disk/bar component formed on a more extended timescale.
\end{abstract}

Key words. stars: abundances - stars: late-type - Galaxy: abundances - Galaxy: bulge - Galaxy: formation

\section{Introduction}

Nearly $25 \%$ of the visible light emitted from stars in the local universe comes from bulges embedded in galactic disks (Fukugita et al. 1998). Therefore, a fundamental step in the

\footnotetext{
* Based on observations collected at the European Southern Observatory, Paranal, Chile (ESO programmes 071.B-0617 and 073.B-0074.

$\star \star$ Full Table 4 is only available at the CDS via anonymous ftp to cdsarc.u-strasbg.fr $(130.79 .128 .5)$ or via http://cdsarc.u-strasbg.fr/viz-bin/qcat?J/A+A/530/A54
}

understanding of galaxy formation and evolution is to obtain an accurate characterization of these structures. The picture seems to be complicated as recent observations suggest that bulges may be an in-homogeneous class of objects, each of them holding a different formation history (e.g. Athanassoula 2005). In this context, The Milky Way bulge is a unique laboratory in which we can study in detail the stellar populations as well as the chemical composition and kinematics on a star-by-star basis (see Minniti \& Zoccali 2008, for a review).

In our galaxy, the consensus is that the bulk of the bulge is old $(t \sim 12 \mathrm{Gyr})$ with a metallicity distribution that extends from 
$[\mathrm{Fe} / \mathrm{H}]=-1.5$ to $[\mathrm{Fe} / \mathrm{H}]=0.5$, with a peak at solar metallicity (e.g. Zoccali et al. 2003, 2008; Clarkson et al. 2008; Brown et al. 2010, and references therein) and that the formation of the bulge was fast as evidenced by the enhanced alpha elements in bulge stars (McWilliam \& Rich 1994; Rich \& Origlia 2005; Zoccali et al. 2006; Fulbright et al. 2006, 2007; Lecureur et al. 2007; Meléndez et al. 2008; Ryde et al. 2009, 2010). A vertical metallicity gradient is also observed (Minniti et al. 1995; Zoccali et al. 2008) although it appears to be absent in the inner bulge regions $\left(b<4^{\circ}\right)$ (Rich et al. 2007). Therefore, the conclusions so far regarding age, metallicity and chemical enrichment in the bulge point to a formation scenario similar to that of early-type galaxies through one or more starburst events triggered by mergers in the early ages of the universe (Weinzirl et al. 2009; Hopkins et al. 2010) - in other words, as a classical bulge.

On the other hand, Kormendy (1993) presented observations of a large number of bulges with properties resembling more disk-like structures rather than the spheroidal concept of classical bulges (see Kormendy \& Kennicutt 2004, for a review). These structures, named pseudo-bulges or disky bulges, tend to show younger stellar populations, to be rotation supported, and to have surface brightness profiles similar to those of disks and less concentrated than those of classical bulges. They are expected to be formed from gas inflows driven by disk instabilities such as the presence of a central bar, which are common phenomena in spiral galaxies. Galaxies, when observed edgeon, often show a central component which swells out of the disk with a boxy, peanut, or even an X shape (e.g. Bureau et al. 2006; Patsis et al. 2002). Several numerical simulations have shown that the inner parts of a bar might evolve and buckle off the plane of the disk showing these particular shapes (Athanassoula 2005; Debattista \& Williams 2004; Debattista et al. 2006).

The above scenarios for bulge formation have historically emerged from the observation of bulges in nearby galaxies. However, a new paradigm has recently emerged, mostly driven by the observations of galaxies at redshift $\sim 2$, i.e., at a lookback time comparable to the age of bulge and thick disk stars. Such galaxies may offer examples of how our own Milky Way was looking like in its early days, prior and during its bulge formation. At $z \sim 2$ a widespread population exists of large, rotating disk galaxies, with much higher gas fractions and velocity dispersion compared to local spirals (Genzel et al. 2006, 2008; Förster Schreiber et al. 2009; Tacconi et al. 2010; Daddi et al. 2010). Such gas-rich galaxies are prone to disk instabilities that can result in bulge formation over timescales of a few $10^{8} \mathrm{yr}$, much shorter than those typically ascribed to secular instabilities in local disk galaxies (Immeli et al. 2004; Carollo et al. 2007; Elmegreen et al. 2008). Such rapid formation, partly due to the quasi-exponential mass growth experienced by these galaxies, quite naturally leads to an $\alpha$-element enhancement in the resulting stellar populations (Renzini 2009; Peng et al. 2010), that would apply both to the disk and bulge stars formed at these early cosmic epochs.

While the existence of a metallicity gradient in the bulge, as shown by Zoccali et al. (2008) strongly argues against the pseudo-bulge hypothesis of Kormendy \& Kennicutt (2004), some recent chemical abundance studies and morphological signatures have provided supporting evidence for such a scenario. Using a sample of high resolution spectra for disk and bulge giants, Meléndez et al. (2008) find a similarity between the $[\mathrm{O} / \mathrm{Fe}]$ ratio of the bulge and the thick disk, both enhanced when compared to the thin disk. Later on, Alves-Brito et al. (2010) reach the same conclusions when analyzing other $\alpha$-elements from the sample of Meléndez et al. (2008). These results are in disagreement with previous results which show a bulge more enhanced in alpha elements than the thick disk. Since those previous studies were done comparing bulge giants to local disk dwarfs from other works (Bensby et al. 2005; Reddy et al. 2006), Meléndez et al. (2008) claim that differences between thick disk and bulge stars might be explained by systematic errors when comparing dwarfs to giants or relative calibrations between those works. If the similarity between the bulge and thick disk is confirmed with a larger and homogeneous sample, this would allow for a common origin for these two galactic components, during the early life of the Galaxy. However, it is important to recall that the origin of the thick disk is also unknown, though several hypothesis have been explored (Di Matteo et al. 2011, and references therein), and most of the results are based on stars from the solar neighbourhood. Progress on this point has been achieved for the first time by Bensby et al. (2010) who provided a sample of inner disk stars which show no significant chemical differences with respect to local disk giants.

In terms of morphology, the evidences for a barred structure in the Milky Way are numerous. Red clump stars distributions from photometric surveys have established the existence of a bar inclined with respect to the Sun-Galactic center line of sight with its near end towards positive longitudes. Observations of the bulge are usually modeled with a triaxial bulge with a position angle of $\sim 15-30$ degrees and nearly $2.5 \mathrm{kpc}$ in length (e.g. Babusiaux \& Gilmore 2005; Rattenbury et al. 2007). Howard et al. (2009) analyzed the kinematics of the bulge concluding that the dynamical signature of the stars at high latitudes $\left(b=-8^{\circ}\right)$ is the same as that in fields closer to the galactic plane $\left(b=-4^{\circ}\right)$, therefore showing evidence for cylindrical rotation as expected for boxy bulges. Their data lack evidence indicative of an accretion origin of bulge in contrast with the claims of tidal streams in kinematics and metallicity from Rangwala \& Williams (2009). Moreover their cylindrical rotation signal was modelled by Shen et al. (2010) without any need for a classical component.

There has also been evidence, from both observations and models, that different types of bulges could coexist in the same galaxy (Samland \& Gerhard 2003; Nakasato \& Nomoto 2003; Kormendy \& Kennicutt 2004; Peletier et al. 2007). If this is the case, we might have a very complex structure in the bulge, with two or more components partially overlapping.

Evidence for a dual nature of our bulge has most recently been invoked based on chemical abundances and kinematic studies. Indication for bimodality in the metallicity distribution has been found from red clump stars in Baade's Window (Hill et al. 2011, submitted). The two components seem to have also different kinematics (Babusiaux et al. 2010) which is consistent with different structures present in the bulge: a metal-rich with barlike kinematics and a metal-poor one showing kinematics of a spheroidal component.

The picture is further complicated by the recent finding of a double peaked red clump along the minor axis, at both positive and negative latitudes, but only for $|b|>6^{\circ}$. The double clump is clearly visible in several color magnitude diagrams available in the literature (e.g. Zoccali et al. 2003; Rattenbury et al. 2007; McWilliam \& Zoccali 2010; Nataf et al. 2010). Such a feature has been interpreted as two components at different distances and is likely to be the outcome of an X-shaped bulge (McWilliam \& Zoccali 2010).

All these observational evidences have suggested a very complex structure of the bulge, with two or more distinct components partially overlapping. Signatures of the different formation histories for these components, should still remain in bulge stars. 
Table 1. Characteristics of our bulge fields.

\begin{tabular}{cccccc}
\hline \hline $\mathrm{N}$ & Name & $l^{\circ}$ & $b^{\circ}$ & $E(B-V)$ & Stars \\
\hline 1 & Baade's Window & 1.14 & -4.18 & 0.55 & 194 \\
2 & $b=-6^{\circ}$ & 0.21 & -6.02 & 0.48 & 194 \\
3 & Blanco field & 0.00 & -12.0 & 0.20 & 86 \\
4 & NGC 6553 & 5.25 & -3.02 & 0.70 & 176 \\
\hline
\end{tabular}

In this paper we analyze the $\alpha$-element distribution of giant stars in three fields along the minor axis and another at a larger galactic longitude. We use them to homogeneously compare with disk giant stars abundances and to investigate the presence of gradients within the bulge.

\section{The sample}

The bulge spectra discussed here belong to the same sample presented in Zoccali et al. (2008, hereafter Z08) as part of a project aimed to provide a spectroscopic characterization of the Galactic bulge. The sample consists of spectra of $650 \mathrm{~K}$ giant stars obtained with the FLAMES-GIRAFFE spectrograph (Pasquini et al. 2003) using the HR13, HR14 and HR15 setups (6100-7000 $\AA$ ) at a resolution of $R \sim 20000$. For the present analysis we have only used the HR13 setup $(R=22500)$ in which we have lines for all the analyzed elements. As described in $\mathrm{Z} 08$, the $\mathrm{S} / \mathrm{N}$ of our sample ranges from 40 to 90 . Galactic coordinates of the bulge fields analyzed here and their extinction values are presented in Table 1 . In the last column of Table 1 we list the number of stars for which we were able to measure abundances for $\mathrm{Ca}, \mathrm{Mg}$, $\mathrm{Ti}$ and $\mathrm{Si}$. These are only those spectra that had sufficient $\mathrm{S} / \mathrm{N}(>50)$ for all the elements to be measured and did not present very large FWHM which could affect the results due to line blending. The sample also contains target stars which belong to bulge globular clusters: NGC 6522 in Baade's Window (7 stars), NGC 6558 in the field at $b=-6^{\circ}$ (3 stars) and NGC 6553 in the eponymous field (29 stars). These stars were clasified as cluster members by Z08 based on the following criteria: $[\mathrm{Fe} / \mathrm{H}]$ within 0.2 dex and radial velocity within $\pm 10 \mathrm{~km} \mathrm{~s}^{-1}$ of the mean for the cluster, and a location within 2 arcmin from the cluster center.

Magnitudes and astrometry for this sample come from the OGLE catalog for Baade's Window and from WFI images for the field at $b=-6^{\circ}, b=-12^{\circ}$ and NGC 6553 (see Z08 for a detailed description). Selected targets are nearly 1 mag above the red clump as shown in Fig. 1.

The sample for thick and thin disk RGB stars in the local neighborhood consists of the same spectra analyzed in Meléndez et al. (2008) and Alves-Brito et al. (2010, hereafter AB10). Stars belong to a range of effective temperatures of $3800<T_{\text {eff }}<$ $5000 \mathrm{~K}$ and metallicities $-1.5<[\mathrm{Fe} / \mathrm{H}]<0.5$, making this sample ideal to compare with our bulge sample. Spectra were taken with different spectrographs, MIKE $(R \sim 65000)$ mounted on the Clay $6.5 \mathrm{~m}$ Magellan telescope, the 2 dcoude $(R \sim 60000)$ spectrograph on the $2.7 \mathrm{~m}$ Harlan J. Smith telescope at McDonald Observatory, HIRES $(R \sim 100000)$ on the Keck I $10 \mathrm{~m}$ telescope and complemented with spectra from the ELODIE archive $(R \sim 42000)$. Population membership and data reduction is described in AB10. Population membership for the bulge sample is described in $\mathrm{Z} 08$.
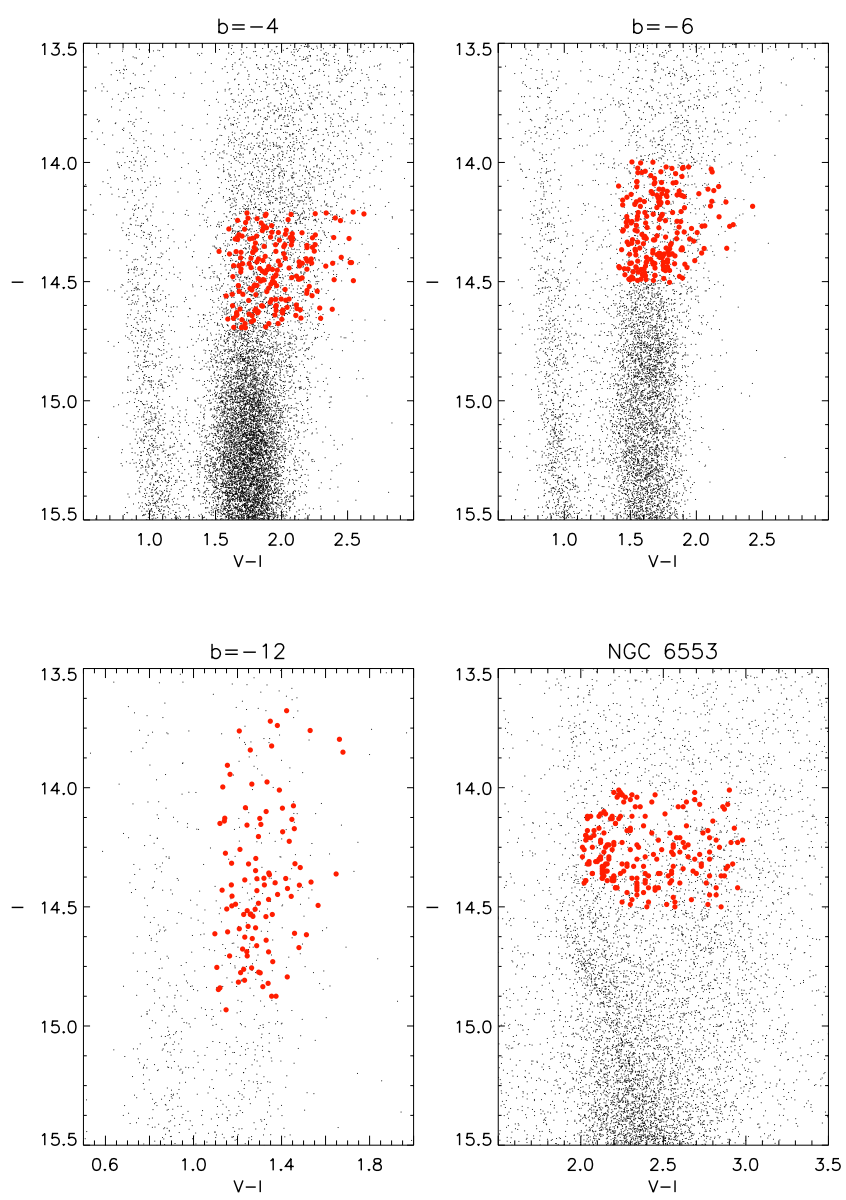

Fig. 1. Color magnitude diagrams for the four fields: Baade's window (upper left), $b=-6^{\circ}$ (upper right), $b=-12^{\circ}$ (lower left) and NGC 6553 (lower right). Spectroscopic targets in each field are marked as large filled circles. Magnitudes were obtained from the OGLE catalogue (Udalski et al. 2002) and the Z08 catalog obtained from WFI images.

\section{Stellar parameters}

For bulge stars we have adopted the same stellar parameters, effective temperature $\left(T_{\text {eff }}\right)$, surface gravity $(\log g)$, microturbulence velocity $(\xi)$ and metallicity $[\mathrm{Fe} / \mathrm{H}]$ presented in $\mathrm{Z} 08$ and we refer the reader to that work for a detailed description of the procedure. In order to perform a differential analysis when comparing bulge and disk stars, we followed the same procedure from Z08 to determine parameters of the disk sample. We have used the same iron linelist from Z08 based on calibrated $\log g f$ values to match the observed spectra of the metal-rich giant $\mu$-Leonis. Only small difference is that as first input value for $T_{\text {eff }}$ we used photometric values obtained from the calibration provided by Alonso et al. (1999) as opposed to the Ramírez \& Meléndez (2005) calibration adopted by Z08. $T_{\text {eff values were }}$ then refined spectroscopically by imposing excitation equilibrium between FeI lines therefore removing any dependance on the adopted calibration. Microturbulence velocity $\xi$ was calculated by requiring the same $[\mathrm{Fe} / \mathrm{H}]$ for all equivalent widths of FeI lines. Surface gravity was determined photometrically using the following equation:

$$
\begin{aligned}
\log (g)= & \log \left(g_{\odot}\right)+\log \left(\frac{M_{*}}{M_{\odot}}\right) \\
& +0.4\left(M_{\mathrm{Bol}, *}-M_{\mathrm{Bol}, \odot}\right)+4 \log \left(\frac{T_{\mathrm{eff}, *}}{T_{\mathrm{eff}, \odot}}\right)
\end{aligned}
$$


where for the Sun we have used the same values used by Zoccali et al. (2008): bolometric magnitude $M_{\mathrm{Bol}, \odot}=4.72 \mathrm{mag}$, effective temperature $T_{\text {eff } \odot}=5770 \mathrm{~K}$ and surface gravity $\log \left(g_{\odot}\right)=$ 4.44 dex. We have adopted the masses from AB10 which are based on Padova isochrones. Absolute visual magnitudes were obtained assuming distances from Hipparcos parallaxes. Finally, bolometric magnitudes were calculated by applying bolometric corrections from Alonso et al. (1999).

\section{Abundance analysis}

$\mathrm{Mg}, \mathrm{Ca}, \mathrm{Ti}$ and $\mathrm{Si}$ abundances were determined by comparing the observed spectra with synthetic ones created with MOOG (Sneden 1973). MOOG is a FORTRAN code that performs a spectral line analysis and spectrum synthesis assuming local thermal equilibrium. MARCS model atmospheres (Gustafsson et al. 2008) were used for our analysis. These models were created using the stellar parameters provided in $\mathrm{Z} 08$ for bulge stars, while for disk stars we used the stellar parameters determined as described in Sect. 3. We have used the same atomic line list from the analysis of bulge stars in Lecureur et al. (2007, hereafter L07). Unfortunately, the lower resolution of GIRAFFE compared to that of the UVES spectra of L07 prevents us to obtain accurate oxygen abundance measurements from the available OI line at $6300 \AA$.

The broad ( $\sim 5 \AA$ ) autoionization Ca I line at $6318.1 \AA$ affects the continuum of the $\mathrm{Mg}$ triplet as discussed in L07. Therefore, $\mathrm{Ca}$ abundances were determined first in our procedure and then this value was given as an input to determine $\mathrm{Mg}$ abundances. The CN line list from the same work was adopted and calibrated carefully as $\mathrm{CN}$ lines are known to be important for cool giant stars. The TiO molecular line list (Plez 1998) was also included. Reference solar abundances are from Asplund et al. (2009).

Although this is a differential analysis, in the sense that we determine the stellar parameters and perform the spectral analysis in the exact same way for all stars, it is important to provide abundances of some reference targets using our procedure, therefore establishing our "zero point" in order to be able to contrast our results with the values obtained in other works. For this reason we have also adopted the calibration procedure based on reproducing the observed spectrum of the Sun, Arcturus and $\mu$-Leonis. We have used the optical spectra of these stars that were analyzed in L07 for the same purpose. $\mu$-Leonis spectrum was taken at the Canada-France-Hawaii with the ESPanDOnS spectropolarimeter at a resolution of 80000 . The Arcturus spectrum with a resolution of 120000 comes from the UVES database (Bagnulo et al. 2003). Finally for the Sun we used a UVES optical spectrum ${ }^{1}$. These spectra were convolved to the GIRAFFE resolution and compared with synthetic spectra in a region around the $\mathrm{Mg}, \mathrm{Ca}, \mathrm{Ti}$, and $\mathrm{Si}$ lines analyzed in this work. Table 2 shows the wavelength, excitation potential $\left(\chi_{\mathrm{ex}}\right)$ and oscillator strength $(\log g f)$ of these lines. Model atmospheres were created adopting the same stellar parameters used in L07, $[\mathrm{Fe} / \mathrm{H}]=0.3, T_{\text {eff }}=4540, \xi=1.3, \log g=2.3$ for $\mu$-Leonis, $[\mathrm{Fe} / \mathrm{H}]=-0.52, T_{\mathrm{eff}}=4300, \xi=1.5, \log g=1.5$ for Arcturus and $T_{\mathrm{eff}}=5770, \xi=0.9, \log g=4.42$ for the Sun. Figure 2 shows the comparison between the synthetic and observed spectra for these stars with the abundances listed in Table 3. For the Sun, we only required a 0.05 dex modification in $\mathrm{Ca}$ abundance with respect to our reference values of Asplund et al. (2009) also listed in Table 3. Additionally, AB10 based

\footnotetext{
${ }^{1}$ (http://www.eso.org/observing/dfo/quality/UVES/ -pipeline/solar_spectrum.html)
}

Table 2. Atomic linelist for $\mathrm{Ca}, \mathrm{Mg}$, $\mathrm{Ti}$ and $\mathrm{Si}$.

\begin{tabular}{cccc}
\hline \hline$\lambda(\AA)$ & Element & $\chi_{\mathrm{ex}}$ & $\log g f$ \\
\hline 6318.71 & $\mathrm{MgI}$ & 5.110 & -2.000 \\
6319.23 & $\mathrm{MgI}$ & 5.110 & -2.240 \\
6319.49 & $\mathrm{MgI}$ & 5.110 & -2.680 \\
6166.43 & $\mathrm{CaI}$ & 2.521 & -1.142 \\
6169.04 & $\mathrm{CaI}$ & 2.523 & -0.797 \\
6169.56 & $\mathrm{CaI}$ & 2.526 & -0.478 \\
6312.23 & $\mathrm{TiI}$ & 1.460 & -1.552 \\
6142.49 & $\mathrm{SiI}$ & 5.620 & -1.500 \\
\hline
\end{tabular}

Table 3. Determined abundances for the Sun, Arcturus and $\mu$-Leonis.

\begin{tabular}{ccccc}
\hline \hline Stars & $\mathrm{A}(\mathrm{Ca})$ & $\mathrm{A}(\mathrm{Mg})$ & $\mathrm{A}(\mathrm{Ti})$ & $\mathrm{A}(\mathrm{Si})$ \\
\hline Sun & 6.39 & 7.60 & 4.95 & 7.51 \\
Sun (Asplund et al. $^{a}$ & 6.34 & 7.60 & 4.95 & 7.51 \\
Arcturus & 5.93 & 7.30 & 4.55 & 7.17 \\
$\mu$-Leonis & 6.65 & 8.02 & 5.31 & 7.85 \\
\hline $\mathrm{AB} 10 \mathrm{ZP}$ & 6.32 & 7.65 & 4.83 & 7.60 \\
This work & 6.49 & 7.65 & 5.09 & 7.62 \\
\hline
\end{tabular}

Notes. The last two rows show the zero point abundances adopted by $\mathrm{AB} 10$ and our mean abundances measured for those same disk giants to which they refer. ${ }^{(a)}$ Reference solar abundances from Asplund et al. (2009).
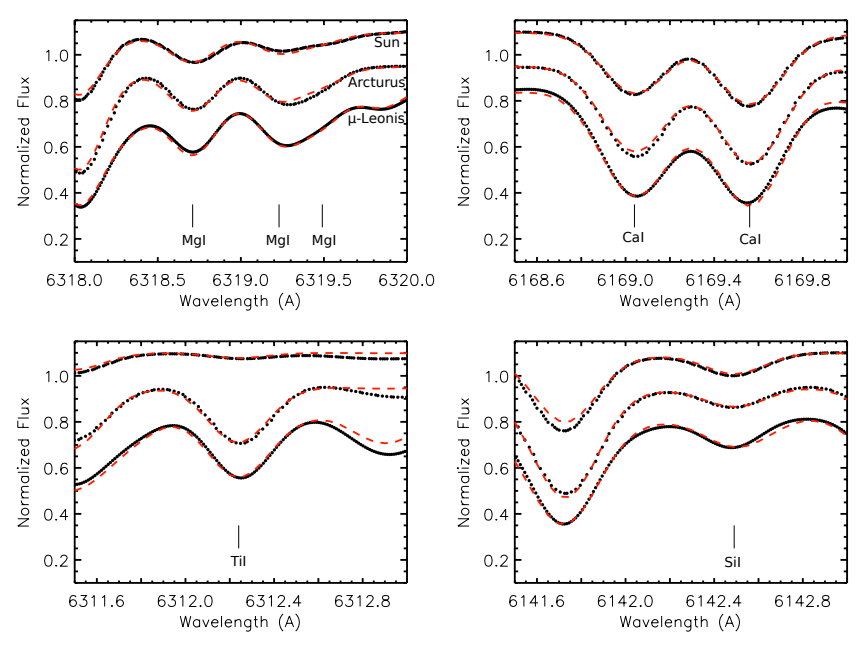

Fig. 2. Calibration of the synthesis regions for the Sun, Arcturus and $\mu$-Leonis. Observed spectra convolved to the GIRAFFE resolution are shown as black circles and the red solid line shows the synthetic spectra using the stellar parameters from L07 and abundances in Table 3.

their zero points in abundances obtained for disk giants with metallicities close to solar value. These zero points are also listed in Table 3 as well as the mean abundances we measure for those same disk giants.

Abundances for our target stars were determined by an iterative process in which the synthetic spectrum is compared to the observed one modifying the abundance in each step of the process until reaching the best fitting value. Given the large number of stars in our sample, we have developed a code which calculates the best fitting abundance by $\chi^{2}$ minimization in a semi-automatic way. In the first step, radial velocities and a first guess for the FWHM of each star is determined using the DAOSPEC code (Stetson \& Pancino 2008). The placement of the continuum is carried out manually for each star, by direct comparison between observed and synthetic spectrum in 

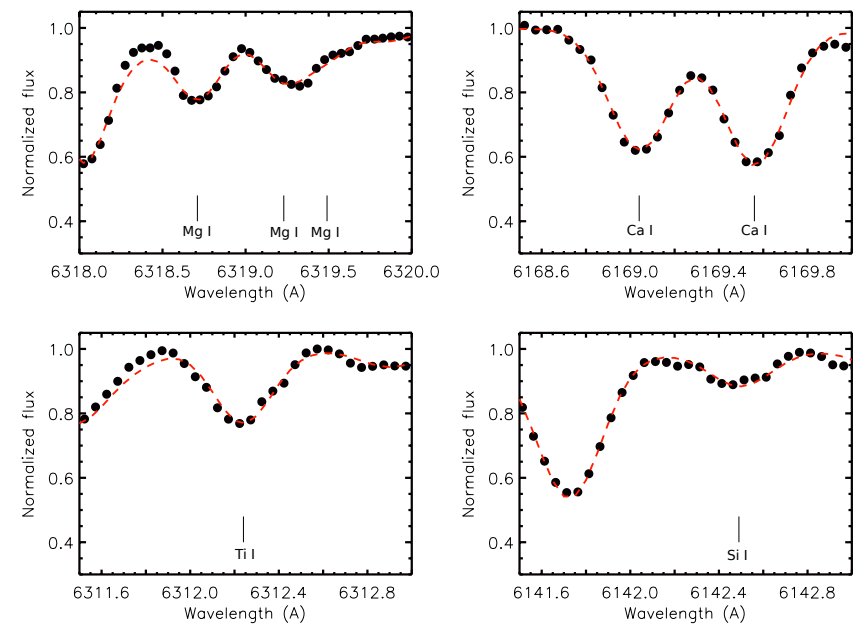

Fig. 3. Example of the final output of the synthesis procedure in order to derive the abundances of $\mathrm{Mg}$ (upper left), $\mathrm{Ca}$ (upper right), Ti (lower left) and $\mathrm{Si}$ (lower right). Observed spectrum for target star 212175c6 is plotted as black dots and the red solid line shows the synthetic spectrum using the best fitting abundance as obtained from our procedure.

a region of $10 \AA$ around the line of interest. Additionally, inspection of nearby lines are used to modify the FWHM from the value obtained from DAOSPEC, when the latter clearly fails to reproduce the spectral features. Later on, the code automatically calculates the best fitting abundance by minimization of a $\chi^{2}$ value which is obtained in the following way:

$\chi^{2}=\Sigma_{\lambda}\left(F_{\text {syn }}(\lambda)-F_{\text {obs }}(\lambda)\right)^{2} \cdot W(\lambda)$

where $F_{\text {syn }}(\lambda)$ is the flux of the synthesis at each wavelength, $F_{\text {obs }}(\lambda)$ is the observed flux previously normalized and $W(\lambda)$ is a weight value calculated as the difference between a normal synthesis and another without the line of the element to be fitted. This allows us to give more importance to the core of the line under analysis. To create this weight we have used $\mu$ Leonis parameters and abundances as a reference (Gratton \& Sneden 1990). Figure 3 shows an example of the final output from the code for each analyzed line.

\section{Error analysis}

\subsection{Error due to stellar parameters uncertainties}

We have carefully analyzed the effect of the different sources of error in our procedure. When obtaining stellar abundances the errors are commonly dominated by the uncertainties in the stellar parameters. Their influence can be determined by varying the adopted parameters by their respective uncertainty and then re-calculating the abundance value which best fits the observed spectra. We have carried out this analysis in a subsample of stars which covers the whole range of metallicities and effective temperatures of our sample. We have used the uncertainties in stellar parameters presented in $\mathrm{Z} 08$ of $200 \mathrm{~K}$ for $T_{\text {eff }}$, 0.3 dex in $\log g, 0.2$ dex in $\xi$ and 0.1 dex in the final $[\mathrm{Fe} / \mathrm{H}]$ value. Figure 4 shows the variation in determined abundances for each element when varying the stellar parameters by their respective uncertainty. The final errors $\sigma_{T}, \sigma_{[\mathrm{Fe} / \mathrm{H}]}, \sigma_{\log g}, \sigma_{\xi}$ associated to each parameter were calculated as the average between the mean changes in abundance when uncertainties were added $\left(\sigma_{(+)}\right)$and subtracted $\left(\sigma_{(-)}\right)$.

Figure 4 shows that errors in the abundance determination are strongly dominated by the uncertainties in the effective temperature determination. Given the adopted uncertainty of $200 \mathrm{~K}$ in temperature, $\mathrm{Mg}$ seems to be the least sensitive element with an associated error of $\sigma_{\Delta T}=0.05$ dex. Ca and $\mathrm{Si}$ show an associated error of 0.07 and 0.10 dex, respectively, and $\mathrm{Ti}$ has the largest dependence on temperature with an error value of $\sigma_{\Delta T}=0.17$ dex. From the lower left panel of Fig. 4 we see that the errors associated to the uncertainties in metallicity are quite similar among the elements with $\sigma_{[\mathrm{Fe} / \mathrm{H}]}$ values ranging between 0.05 and 0.10 dex for an adopted 0.1 dex error in $[\mathrm{Fe} / \mathrm{H}]$. Contrary to the sensitivity of the other elements, $\mathrm{Mg}$ is more affected by changes in metallicity than in temperature. The reason for such a dependence is most likely given by the blend with the strong FeI line at the red side of the triplet. Additionally Fig. 4 shows that the errors arising from uncertainties in gravity, $\sigma_{\log g}$, and microturbulence, $\sigma_{\xi}$, are small. However, Si still shows a 0.05 dex variation given an uncertainty of 0.3 dex in gravity. This is an important point to notice given that in our analysis we assume a distance of $8 \mathrm{kpc}$ for all bulge stars, neglecting the bulge distance spread. If such stars are actually at $6 \mathrm{kpc}$, the bolometric magnitude will be underestimated by $0.65 \mathrm{mag}$. Such an error will change the photometric gravity by 0.25 dex. $\mathrm{Mg}$ abundances are almost completely in-sensitive to changes in both $\log g$ and $\xi$ while $\mathrm{Ca}$ and $\mathrm{Ti}$ show a dependence of 0.06 dex on a microturbulence change of 0.2 dex.

\subsection{Errors from the spectral synthesis}

As described in Z08, stars in the field at $b=-12$ were observed twice due to a mistake in the fiber allocation. For this reason we only have about half the number of stars than in the other fields. However, we can use this repeated observation to carry out the same procedure to re-derive the abundances for all the elements analyzed in our work. In this way, we can estimate the influence of the steps in our analysis which are done by eye such as continuum placement and FWHM adjustment used for the synthesis. Additionally this will also provide an estimate for the errors associated to different $\mathrm{S} / \mathrm{N}$ among stars in the same field. Figure 5 shows the difference between the two independent analysis. The mean value of the difference for all elements ranges between 0 and 0.02 dex, which shows the absence of systematics in our procedure. The scatter is quite constant among the elements up to a value of 0.1 dex. Since the stellar parameters were not recalculated, this scatter is not produced by their uncertainties but from the procedure to measure the abundances. We can therefore assume a 0.1 dex error from this source, which added to the errors from the stellar parameters gives an estimation for the final errors in our measured abundances following the relation:

$\sigma_{[\mathrm{X} / \mathrm{Fe}]}^{2}=\sigma_{\Delta T}^{2}+\sigma_{\Delta[\mathrm{Fe} / \mathrm{H}]}^{2}+\sigma_{\Delta \log g}^{2}+\sigma_{\Delta \xi}^{2}+\sigma_{\mathrm{syn}}^{2}$

Therefore, the final values of the estimated error in our analysis are 0.15 dex for $[\mathrm{Mg} / \mathrm{Fe}], 0.16 \mathrm{dex}$ for $[\mathrm{Ca} / \mathrm{Fe}], 0.22 \mathrm{dex}$ for $[\mathrm{Ti} / \mathrm{Fe}]$ and $0.14 \mathrm{dex}$ for $[\mathrm{Si} / \mathrm{Fe}]$.

\subsection{The role of spectral resolution}

It is also necessary to consider the effect of using lower resolution $(R \sim 22500)$ spectra from GIRAFFE in comparison to the high resolution of the disk sample of $R>45000$. In order to address this question we have re-calculated all the abundances using high resolution spectra for a subsample of stars in each field that was also observed with UVES $(R \sim 45000)$. The abundances were calculated following the same procedure as for the GIRAFFE sample. 

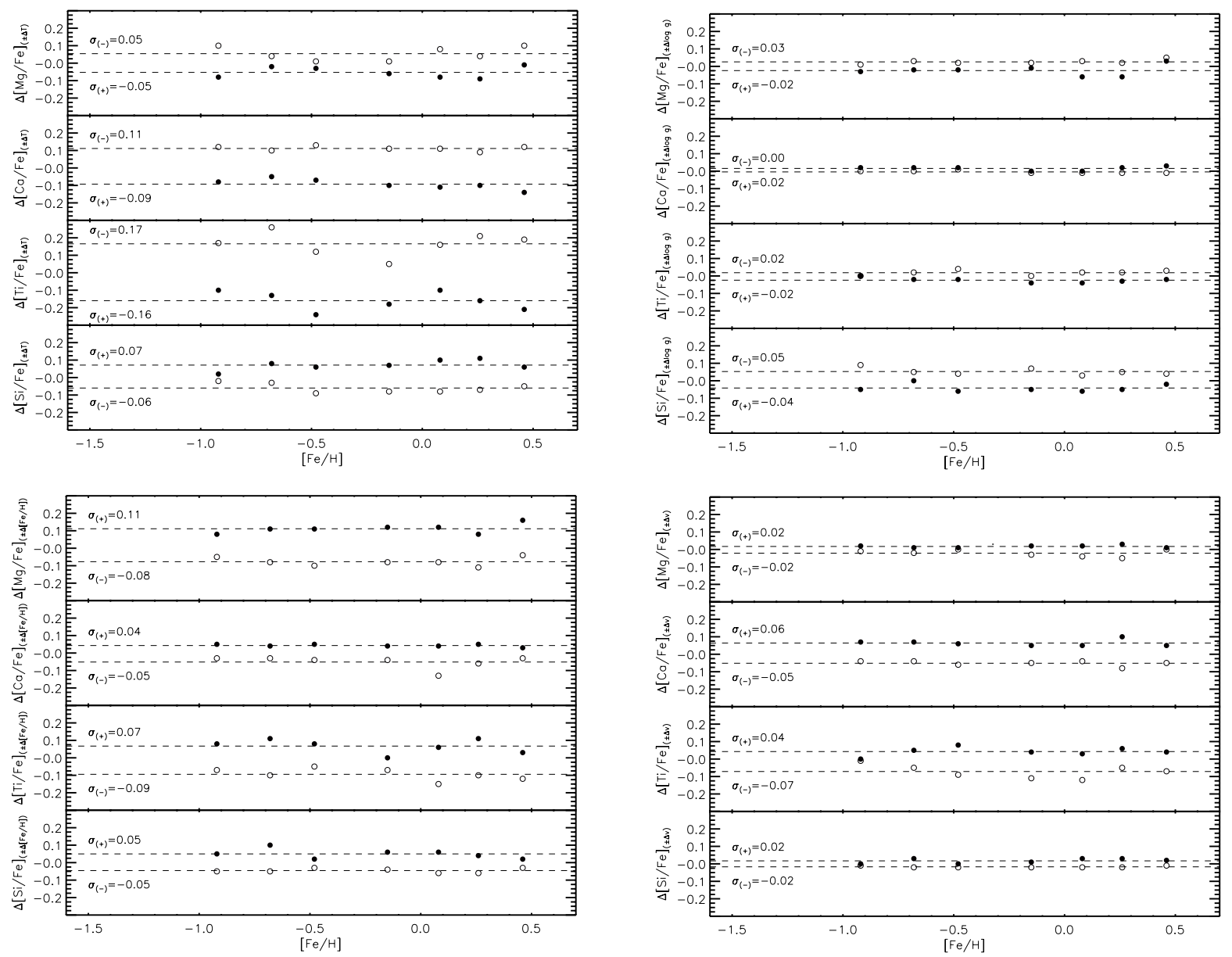

Fig. 4. Abundance errors associated with the uncertainties in effective temperature (upper left), surface gravity (upper right), metallicity (lower left) and microturbulence (lower right). A change of $\pm 200 \mathrm{~K}$ was applied to $T_{\text {eff }}, \pm 0.1$ dex to $[\mathrm{Fe} / \mathrm{H}], \pm 0.3$ dex to $\log g$ and \pm 0.2 dex to $\xi$. Model atmosphere were then created and abundances were re-determined. Filled circles show the change in abundance as $\Delta[\mathrm{X} / \mathrm{Fe}]=[\mathrm{X} / \mathrm{Fe}]-[\mathrm{X} / \mathrm{Fe}]_{ \pm \Delta}$ when changes were added to the original parameters. Empty circles show the change in abundance when the respective value is subtracted. Dashed lines show the mean variations $\sigma_{(+)}$and $\sigma_{(-)}$when the uncertainty in the stellar parameter is added and subtracted respectively.

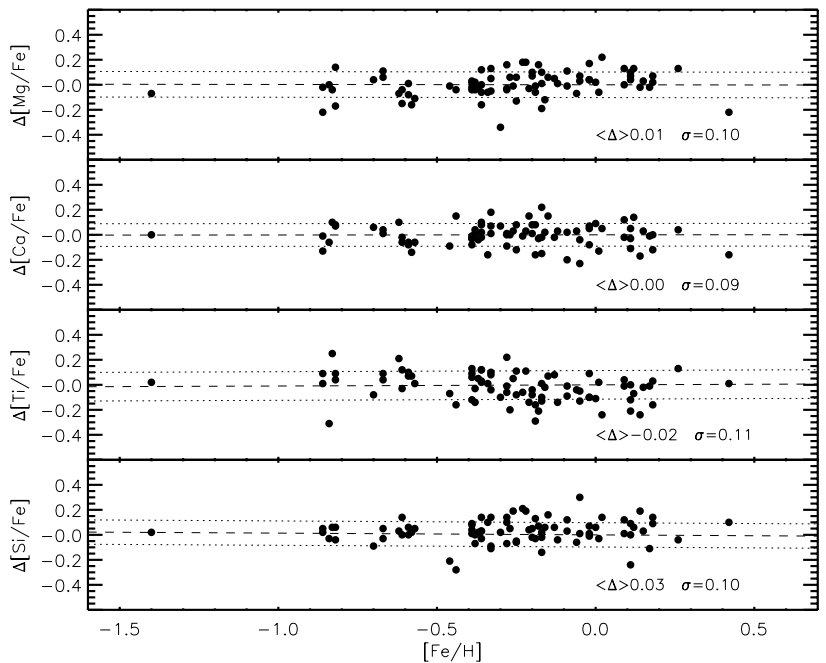

Fig. 5. Difference between the abundances measured using the same lines for stars observed twice in the field at $b=-12$. The dashed line shows the mean difference between both measurements and the dotted lines the $1 \sigma$ around the mean.

Figure 6 shows the comparison between the abundances for each star in all analyzed elements. No systematic offsets are

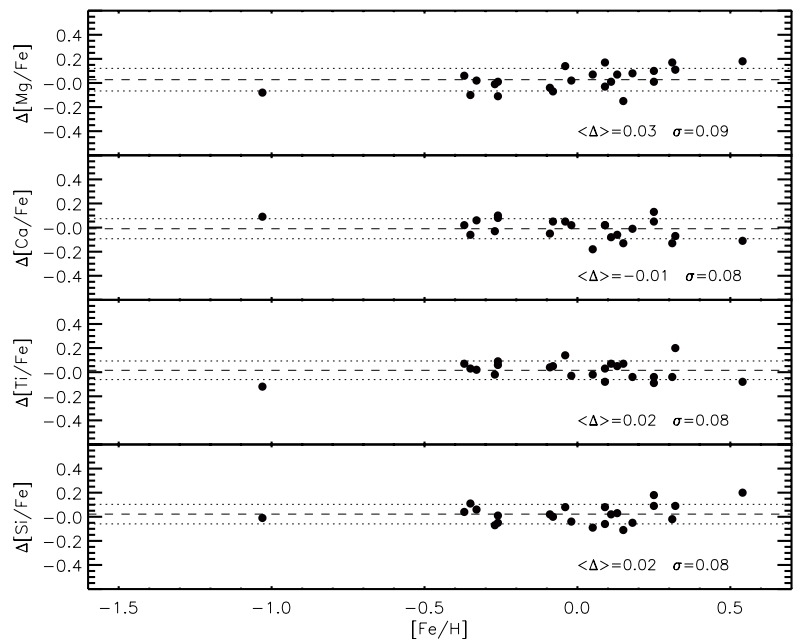

Fig. 6. $\Delta[\alpha / \mathrm{Fe}]=[\alpha / \mathrm{Fe}]_{\text {UVES }}-[\alpha / \mathrm{Fe}]_{\text {GIRAFFE }}$ for our bulge sample plotted as black filled circles. The dashed line indicates the mean value of the difference and the dotted lines show the $1 \sigma$ range around the mean difference for each element.

observed in our analysis from the change in resolution. This is particularly important to notice since it could be a source of systematic shifts in the trends when comparing bulge and disk. 
O. A. Gonzalez et al.: Galactic bulge $\alpha$-element abundances

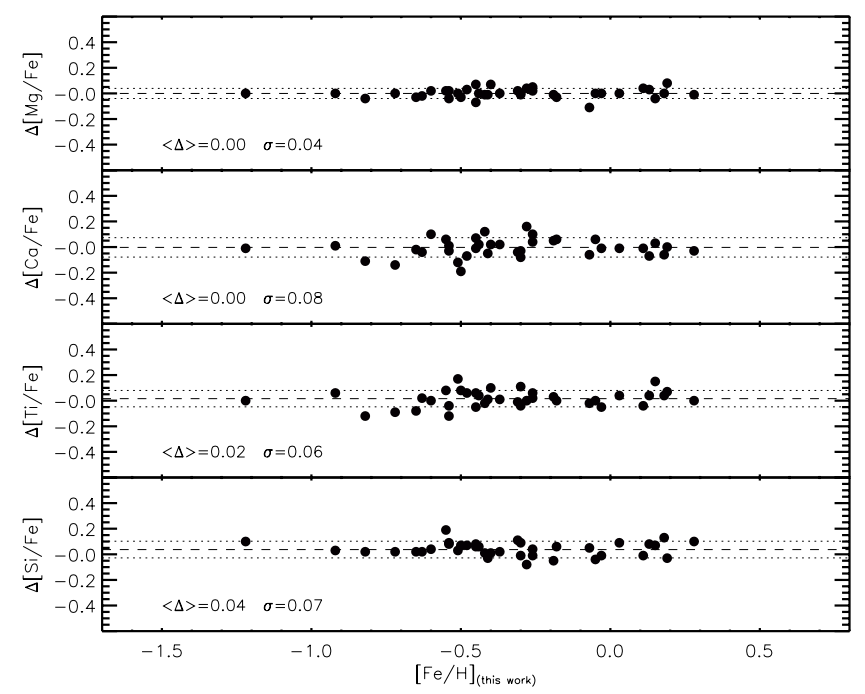

Fig. 7. $\Delta[\alpha / \mathrm{Fe}]=[\alpha / \mathrm{Fe}]_{\mathrm{HR}}-[\alpha / \mathrm{Fe}]_{\mathrm{LR}}$ for the disk sample plotted as black filled circles where the low resolution (LR) spectra were obtained by degrading the high resolution (HR) to the resolution of our GIRAFFE sample. The dashed line indicates the mean value of the difference and the dotted lines show the $1 \sigma$ range for each element.

In order to further check the existence of any systematics we have also redetermined the abundances in the disk sample after degrading the resolution of the spectra to the one of GIRAFFE. Figure 7 confirms that no differences are observed from a change in resolution. Only $\mathrm{Si}$ shows a mean difference of $0.04 \mathrm{dex}$ which is negligible within the errors on the abundance itself.

Additionally we have re-measured the abundances in our calibrator stars Arcturus, $\mu$-Leonis and the Sun, using the spectra with the original high resolution. The results were compared with our abundance measurements from convolved spectra (Table 3 and Fig. 2) and no differences were found. We therefore conclude that our comparison of bulge stars based on lower resolution to those of the disk is valid and no systematic shifts should be applied to our results.

\section{Results}

\subsection{Thick and thin disk sample}

In order to characterize the bulge alpha abundances and compare them to other galactic components we have checked carefully the homogeneity of the procedure. Stellar parameters for the disk sample were calculated following the same procedure described in Z08. A comparison between the stellar parameters obtained using our procedure and the values presented in AB10 is shown in Fig. 8. We systematically find a larger effective temperature with a mean difference of $130 \mathrm{~K}$ between the two methods. Also, $\log g$ values are systematically larger in our analysis with respect to AB10. These differences between our studies show once more the importance of a homogeneous analysis in which parameters for all samples are on the same scale.

Using our determination of the stellar parameters, we have measured the abundances for $\mathrm{Mg}, \mathrm{Ca}, \mathrm{Ti}$ and $\mathrm{Si}$ as described in Sect. 3. Figure 9 shows the difference between the abundances for the disk sample derived in this work and the values presented in AB10. Mg abundances appear to be on the same scale however abundances of $\mathrm{Ca}$ and $\mathrm{Ti}$ show larger differences.

AB10 uses the abundances of a subsample of thin disk stars, with metallicity near to the solar value, as internal zero-points.
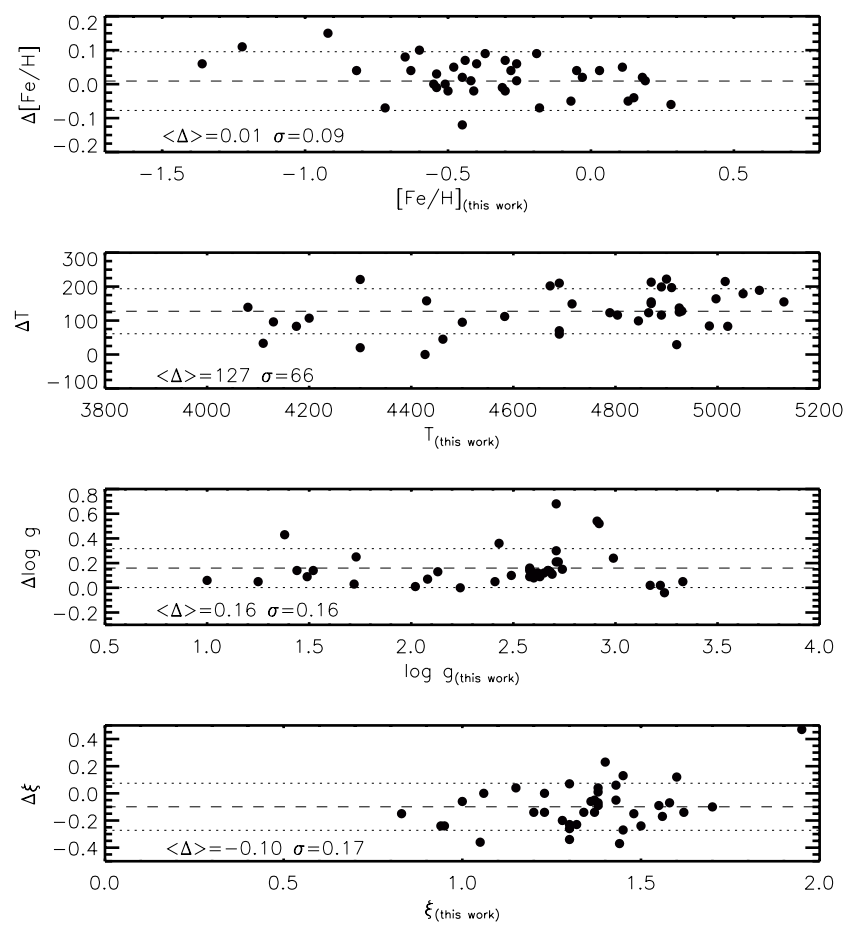

Fig. 8. Difference between the stellar parameters obtained in this work and the values presented in $\mathrm{AB} 10$ calculated as $\Delta X=X_{\text {this work }}-X_{\mathrm{AB} 10}$. The dashed line indicates the mean value of the difference and the dotted lines show the $1 \sigma$ range for each element.

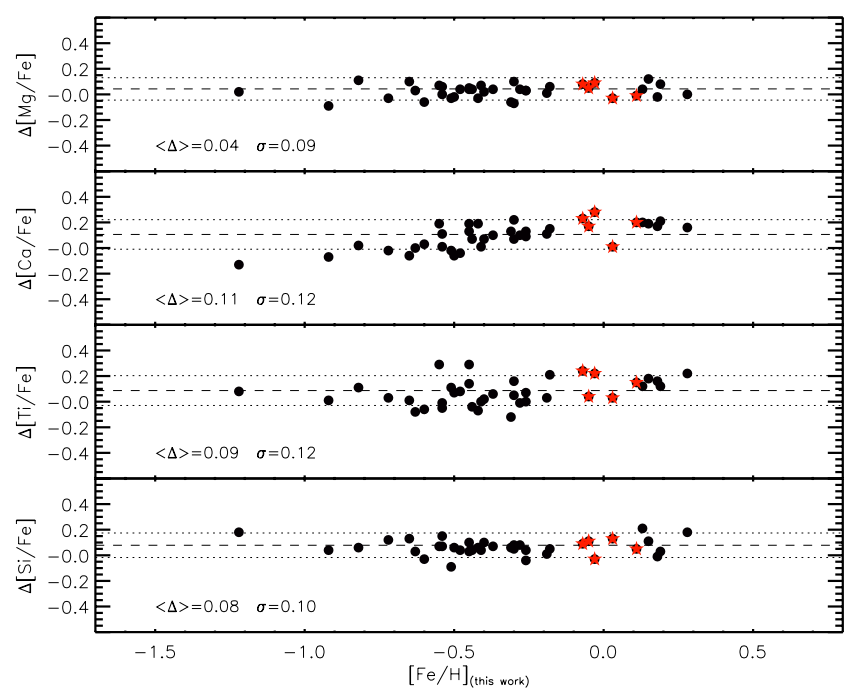

Fig. 9. Difference between the $[\alpha / \mathrm{Fe}]$ values for $\mathrm{Mg}, \mathrm{Ca}, \mathrm{Ti}$ and $\mathrm{Si}$ obtained in this work and the values presented in $\mathrm{AB} 10$ calculated as $\Delta[\alpha / \mathrm{Fe}]=[\alpha / \mathrm{Fe}]_{\text {this work }}-[\alpha / \mathrm{Fe}]_{\mathrm{AB} 10}$. The dashed line indicates the mean value of the difference and the dotted lines show the $1 \sigma$ range for each element. In particular, disk giants used by AB10 to calculate their internal zero points are shown as red filled stars.

For the same stars we find larger $\mathrm{Ca}$ and $\mathrm{Ti}$ abundances as shown in Table 3. However we remain with our zero-points based on the solar abundances of Asplund et al. (2009). Table 3 should be used to compare our results either with models scaled to solar abundances or other works on different scales. Within our $\log g f$ and adopted zero points, the homogeneity of our analysis remains unchanged. 
Table 4. $\mathrm{Mg}, \mathrm{Ca}$, Ti and $\mathrm{Si}$ abundances for stars in our bulge fields.

\begin{tabular}{ccccccc}
\hline \hline Star ID & {$[\mathrm{Fe} / \mathrm{H}]$} & {$[\mathrm{Mg} / \mathrm{Fe}]$} & {$[\mathrm{Ca} / \mathrm{Fe}]$} & {$[\mathrm{Ti} / \mathrm{Fe}]$} & {$[\mathrm{Si} / \mathrm{Fe}]$} & Field \\
\hline 423342 & +0.46 & -0.04 & +0.13 & +0.03 & -0.08 & 1 \\
423323 & -0.48 & +0.43 & +0.15 & +0.23 & +0.26 & 1 \\
412779 & -0.37 & +0.23 & +0.29 & +0.48 & +0.22 & 1 \\
423359 & -1.23 & +0.34 & +0.30 & +0.48 & +0.43 & 1 \\
$\ldots \ldots$ & $\ldots .$. & $\ldots .$. & $\ldots .$. & $\ldots$. & $\ldots .$. & $\ldots \ldots$ \\
\hline
\end{tabular}

Notes. Metallicities are those presented in Z08 and listed here for reference. The full table is available in electronic form at the CDS.

\subsection{Alpha elements across the bulge, thick and thin disk}

The final $[\alpha / \mathrm{Fe}]$ trends for $\mathrm{Mg}, \mathrm{Ca}, \mathrm{Ti}$, and $\mathrm{Si}$ are shown in Figs. 10 to 13 and listed in Table 4. The general alpha enhancement of the bulge stars is observed clearly in all four elements. The alpha enhancement is also seen in our globular cluster members and their mean abundances do not differ from those of field stars at their given metallicity. The scatter in the observed trends is different: $\mathrm{Mg}$ abundances show much smaller scatter than $\mathrm{Ca}$, $\mathrm{Ti}$ and $\mathrm{Si}$ abundances. In particular at low metallicities, where there is almost no dependence on $[\mathrm{Fe} / \mathrm{H}]$, there is a $\sim 0.09$ dex scatter observed for $[\mathrm{Mg} / \mathrm{Fe}]$ and $\sim 0.13 \mathrm{dex}$ for the other elements. As shown in Sect. 5, the dependence of the measured abundance on the stellar parameter uncertainties also varies among elements and the observed scatter in abundances is within the estimated errors. Therefore, the different amount of scatter is largely due to the uncertainties in the parameters. The similarity and overabundance in the bulge and thick disk stars appears to vary among the elements and therefore trends should be compared separately.

$\mathrm{Mg}$ is the element which shows the best defined trend among the elements analyzed here. This element shows the least dependency on the stellar parameters and can be considered as the most reliable of the alpha elements for chemical enrichment studies as, together with oxygen, it is expected to be enriched exclusively by SNII explosions. The mean abundance of the bulge at $[\mathrm{Fe} / \mathrm{H}]<-0.5$ is $[\mathrm{Mg} / \mathrm{Fe}]=+0.31$ which only differs by 0.03 dex from the mean thick disk at the same metallicity range with the bulge being slightly more enhanced than the thick disk. Such a behavior remains in the intermediate metallicity range of our sample $(-0.5<[\mathrm{Fe} / \mathrm{H}]<-0.2)$ where the bulge shows a mean $[\mathrm{Mg} / \mathrm{Fe}]$ of 0.27 and is still only 0.03 dex more enhanced that the thick disk. The differences we have found between these populations are much smaller than those found by L07, in which the bulge is up to 0.2 dex more enhanced than the sample of thick disk dwarfs from the literature. Further comparison at higher metallicities are difficult as separation between thin and thick disk becomes difficult. All elements show that, at the high metallicity range $([\mathrm{Fe} / \mathrm{H}]>0)$ both the bulge and two of the high metallicity stars classified as thick disk by AB10 have lower ratios, as low as those of the thin disk.

On the other hand, $[\mathrm{Ca} / \mathrm{Fe}]$ trends show a larger scatter which complicates the comparison, particularly at high metallicities. However, at lower metallicities, where actually the thick disk is well defined, differences in $[\mathrm{Ca} / \mathrm{Fe}]$ between the mean of both populations are higher showing the bulge 0.09 dex more enhanced than the thick disk at $[\mathrm{Fe} / \mathrm{H}]<-0.5$ and 0.04 dex at higher metallicities. Worth notice as well is that $[\mathrm{Ca} / \mathrm{Fe}]$ shows a shallower decline with metallicity than $\mathrm{Mg}$ in good agreement with model predictions where $\mathrm{Ca}$ contribution is also expected from SNIa explosions.

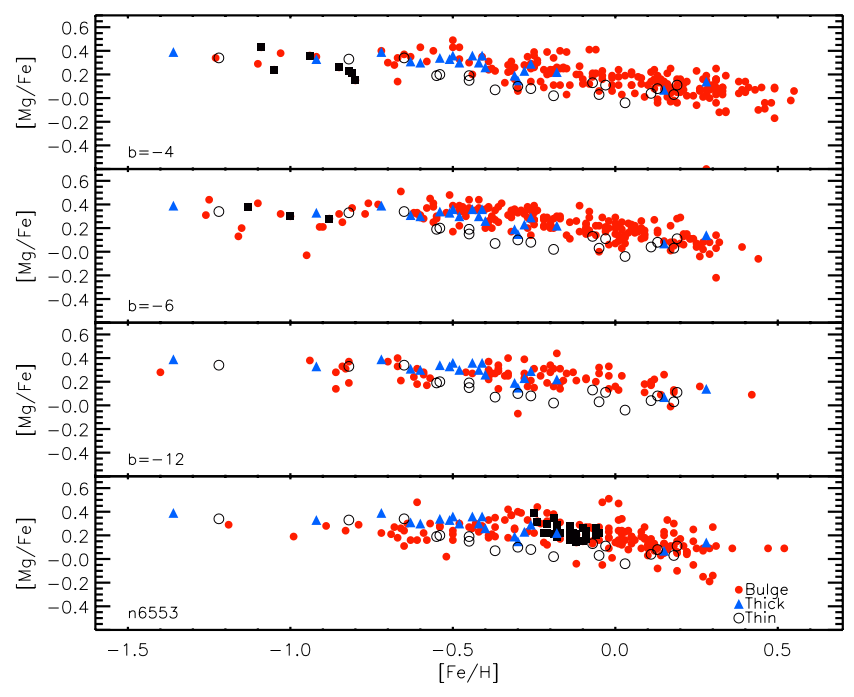

Fig. 10. $[\mathrm{Mg} / \mathrm{Fe}]$ abundances in 4 fields of the bulge shown as red filled circles. Bulge globular cluster members are shown as black filled squares. $[\mathrm{Mg} / \mathrm{Fe}]$ abundances for the thick disk stars are shown as blue filled triangles and as empty black circles for the thin disk stars.

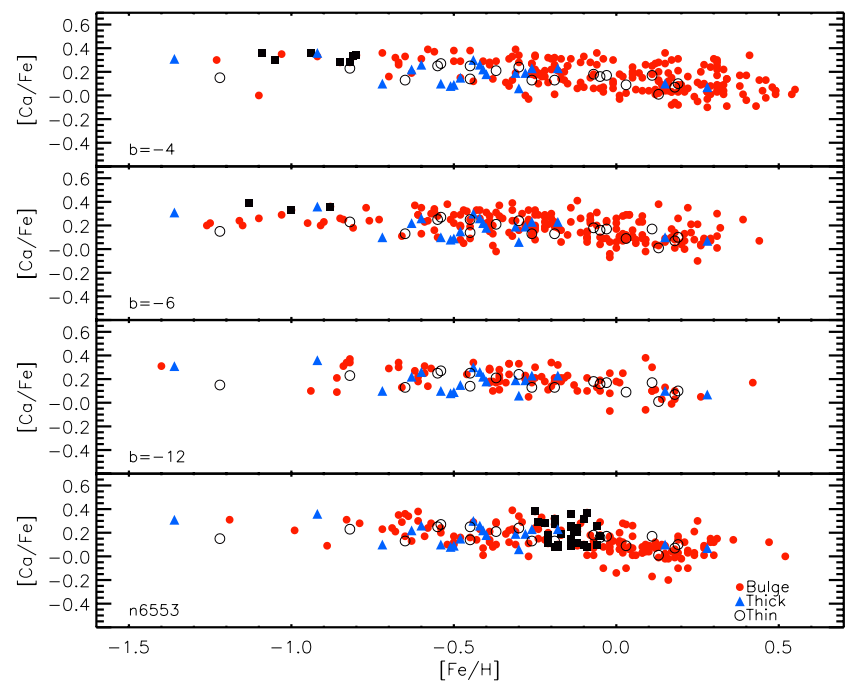

Fig. 11. $[\mathrm{Ca} / \mathrm{Fe}]$ abundances in 4 fields of the bulge. Symbols are as in Fig. 10.

Results for [Ti/Fe] are similar to those observed in $\mathrm{Ca}$, showing trends with a shallower decline with metallicity but also a larger scatter. The mean abundance is $[\mathrm{Ti} / \mathrm{Fe}]=0.40$ and shows a difference with the thick disk of 0.10 dex from $[\mathrm{Fe} / \mathrm{H}]<-0.5$ up to intermediate metallicities. Only above solar metallicities the mean Ti abundances of bulge and thick disk are the same. However these differences are within the scatter of the Ti abundances in the bulge which is consistent with the larger error dependence on temperature. Moreover, the $[\mathrm{Ti} / \mathrm{Fe}]$ ratios in the thick disk are actually within the lower envelope of those of the bulge.

Finally in the case of [Si/Fe], trends for the disk and the bulge are almost undistinguishable as in the case of $\mathrm{Mg}$, with the bulge showing a mean $[\mathrm{Si} / \mathrm{Fe}]=0.35$ at $[\mathrm{Fe} / \mathrm{H}]<-0.5$ and differences lower than 0.03 dex with the thick disk at all metallicities.

Figure 14 shows the average $\alpha$ element abundances. Trends for the bulge and both thin and thick disk are very well defined and we can also use them to compare the population properties between our different fields along the minor axis and with the 


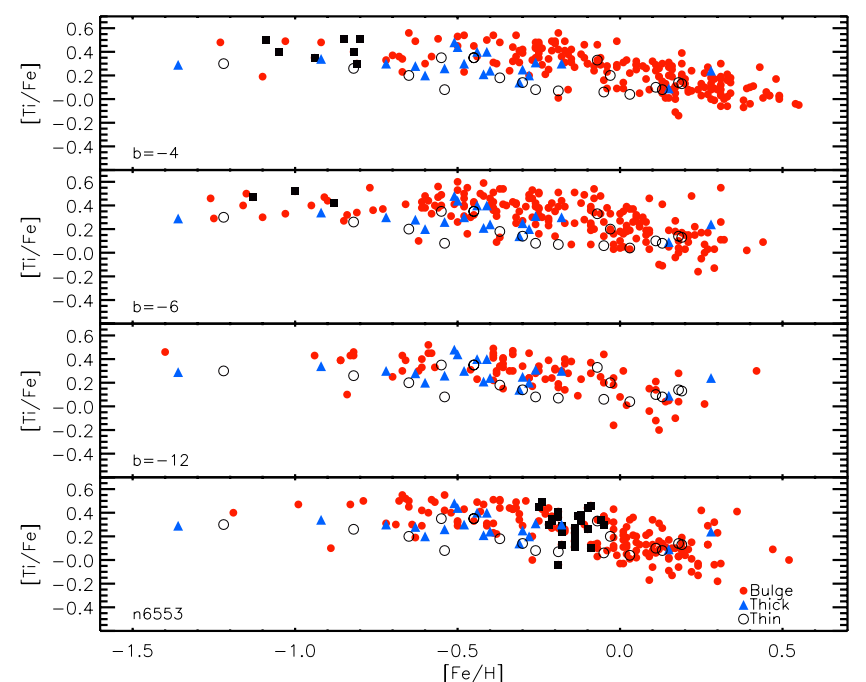

Fig. 12. $[\mathrm{Ti} / \mathrm{Fe}]$ abundances in 4 fields of the bulge. Symbols are as in Fig. 10.

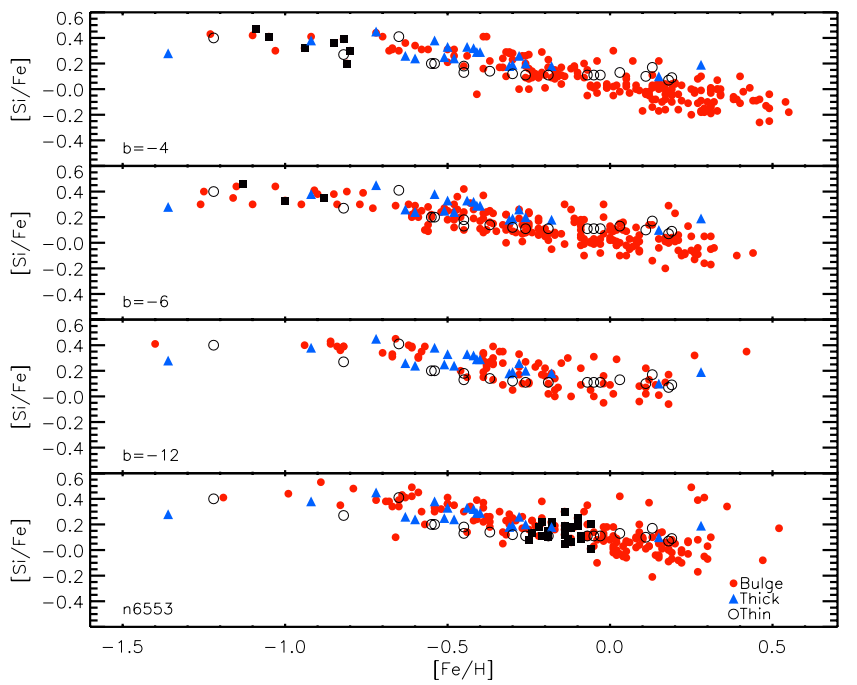

Fig. 13. $[\mathrm{Si} / \mathrm{Fe}]$ abundances in 4 fields of the bulge. Symbols are as in Fig. 10.

field at a larger galactic longitude of $l=5^{\circ}$. A discussion regarding such a comparison is addressed in Sect. 6.3.

In order to generally compare abundances between bulge and disk, we have followed the procedure adopted in AB10 and fit two independent slopes for two different metallicity regimes. However, instead of fixing arbitrarily the point where the knee is produced to a $[\mathrm{Fe} / \mathrm{H}]$ value of -0.5 , as done in $\mathrm{AB} 10$, we have produced a linear fit to a metal-poor region between -1.2 and -0.5 dex and to a metal-rich region between -0.3 and 0.2 dex. These regions where selected in order to i) consider metallicity regions in which the trends are well defined and ii) avoid taking into account too metal-rich stars in which the uncertainties are larger. In this way, we are now able to provide a value for the metallicity at which the knee is actually produced, calculated as the intersection of the linear fits of both regions. The best fitting relations for both metallicity ranges in all the bulge fields and the thick disk are shown in Table 5 and plotted in Figs. 15 and 16.

As a general result, when considering the mean abundances of all the elements analyzed, differences between the bulge and the thick disk do not exceed 0.05 dex for all metallicities. Trends between both populations are therefore indistinguishable within

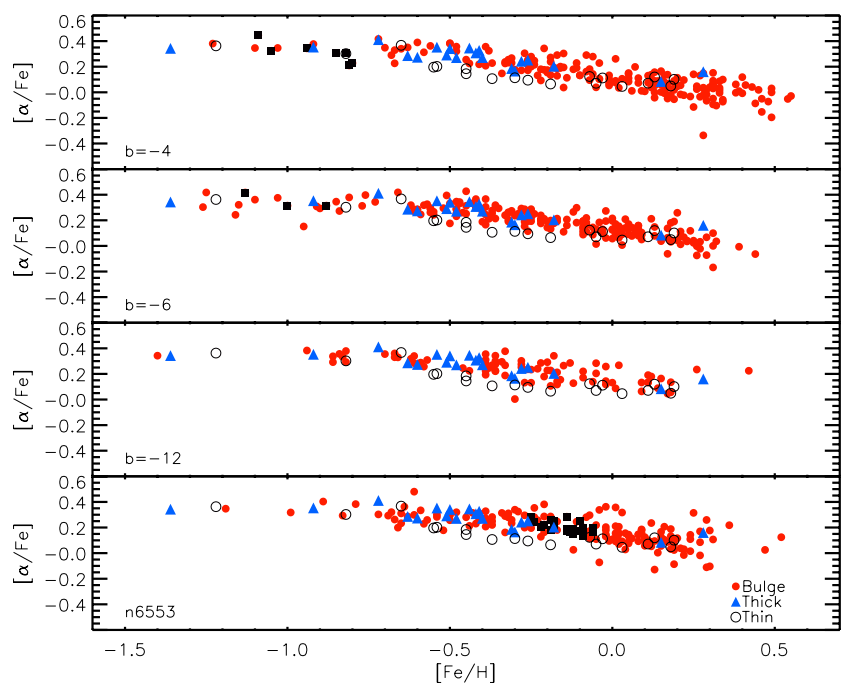

Fig. 14. $[\alpha / \mathrm{Fe}]$ abundances in 4 fields of the bulge calculated as the average between $\mathrm{Ca}, \mathrm{Mg}$, Ti and $\mathrm{Si}$ abundances. Symbols are as in Fig. 10.

the errors of our analysis. Additionally we see that the knee in the bulge in Baade's Window is located at a metallicity of $[\mathrm{Fe} / \mathrm{H}]=-0.44$ and differs by less than 0.1 dex among the other bulge fields. This knee is also present in the thick disk distribution at $[\mathrm{Fe} / \mathrm{H}]=-0.51$, only 0.07 dex different to the location for the observed bulge downtrend. Therefore, within the errors considered in our analysis as discussed in previous sections, no significant differences are observed between the bulge and the thick disk abundances for individual elements. The similarity is even stronger when all elements are compared in the mean.

\subsection{Is there an alpha element gradient in the bulge?}

The existence of a metallicity gradient in the bulge is considered a strong indication towards the classical bulge formation scenario and is not expected to be present in boxy or pseudo-bulges. On the other hand, if Galactic bulge is a mixture of two (or more) components, it could be expected that these populations should have gone through different formation histories. A component formed via mergers would show higher alpha elements than a structure dynamically formed from disk material which would have enough time for SNIa to explode and decrease the $[\alpha / \mathrm{Fe}]$ ratio up to Solar values. These components might have different relative contributions across the bulge and therefore their content of alpha elements might help to distinguish between them.

Figures 15 and 16 show that in the case of the Galactic bulge, the relations between $[\alpha / \mathrm{Fe}]$ and metallicity are undistinguishable among the different fields analyzed here. From the obtained relations for each field and in the two metallicity regimes that we have considered here, we can actually see no significant difference between the fields in terms of the IMF and the timescale given by the knee at which SNIa contribution becomes important. Differences between the fields are not larger than 0.02 dex at all metallicity regimes and the knee is observed at the same metallicity within $0.1 \mathrm{dex}$. This variation is in fact within our estimation for the uncertainties in $[\mathrm{Fe} / \mathrm{H}]$. Such small differences are fairly consistent with the errors considered in our abundance analysis and a striking similarity can be concluded both along the minor axis (Fig. 15) and the major axis (Fig. 16). Also shown in Figs. 15 and 16, are the $[\alpha / \mathrm{Fe}]$ distributions for each of the fields. The behavior of such distributions are consistent with the metallicity gradient in the bulge in which the population of stars 
Table 5. Abundance trends for the bulge and thick disk.

\begin{tabular}{lcccc}
\hline \hline & \multicolumn{2}{c}{$-1.2<[\mathrm{Fe} / \mathrm{H}]<-0.5$} & \multicolumn{2}{c}{$-0.3<[\mathrm{Fe} / \mathrm{H}]<0.2$} \\
Field & $m$ & $a$ & $m$ & $a$ \\
\hline Baade's Window & $-0.07 \pm 0.07$ & $0.27 \pm 0.06$ & $-0.43 \pm 0.04$ & $0.119 \pm 0.006$ \\
$b=-6^{\circ}$ & $-0.06 \pm 0.04$ & $0.27 \pm 0.04$ & $-0.33 \pm 0.04$ & $0.134 \pm 0.006$ \\
Blanco field & $-0.07 \pm 0.04$ & $0.27 \pm 0.03$ & $-0.32 \pm 0.06$ & $0.16 \pm 0.01$ \\
NGC 6553 & $-0.08 \pm 0.09$ & $0.25 \pm 0.06$ & $-0.39 \pm 0.06$ & $0.148 \pm 0.008$ \\
Thick disk & $-0.04 \pm 0.06$ & $0.30 \pm 0.05$ & $-0.37 \pm 0.08$ & $0.13 \pm 0.03$ \\
\hline
\end{tabular}

Notes. Best fit parameters were calculated for linear $(a+m[\mathrm{Fe} / \mathrm{H}])$ trends for $[\langle\mathrm{Mg}, \mathrm{Ca}, \mathrm{Ti}, \mathrm{Si}\rangle / \mathrm{Fe}]$ abundances in the bulge and thick disk.
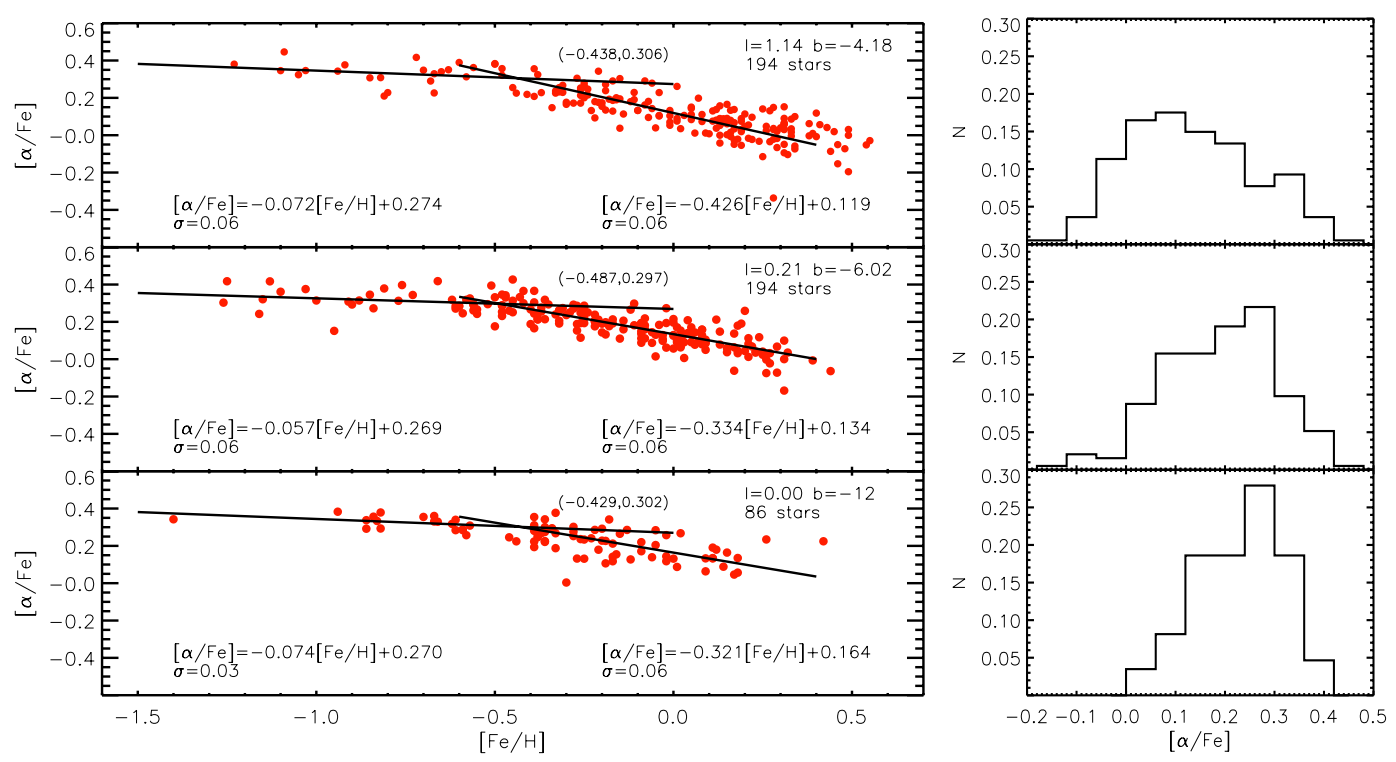

Fig. 15. The left panels show $[\alpha / \mathrm{Fe}]$ trends as a function of $[\mathrm{Fe} / \mathrm{H}]$ in 3 bulge fields located along the minor axis. Best fit trends are shown for both $[\mathrm{Fe} / \mathrm{H}]$ ranges, a metal-poor between -1.2 and -0.5 dex and a metal-rich between -0.3 and 0.2 dex, as well as the location of the knee in all fields. The right panels show the $[\alpha / \mathrm{Fe}]$ distribution for each field.
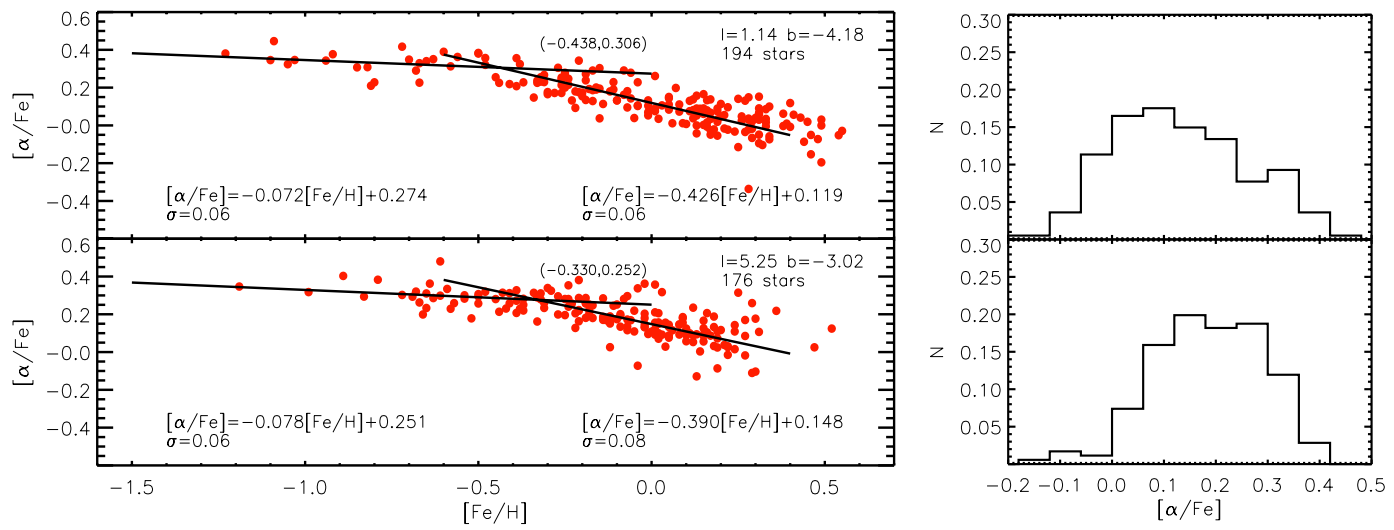

Fig. 16. Same as in Fig. 15 for 2 bulge fields along the major axis.

with higher metallicities $([\mathrm{Fe} / \mathrm{H}] \sim 0)$ and lower $\alpha$ element abundances $([\alpha / \mathrm{Fe}] \sim 0)$ becomes smaller at higher distances from the galactic plane.

\subsection{Correlation with kinematics}

Coupling the information from radial velocities, proper motions and metallicity for the same bulge FLAMES sample that we present here, Babusiaux et al. (2010) demonstrate that metalrich component shows disk/bar kinematics and a metal-poor component has kinematics of an old spheroid. The formation time-scale for the old (and metal-poor) spheroid component is much shorter, than that of the disk/bar component, and therefore it is expected that the former is alpha enhanced, while the latter has lower alpha element abundance. We investigate this by combining our alpha element abundances with radial velocity information from Z08. Figure 17 shows the radial velocity dispersion for different alpha element abundance bins. We observe that alpha-enhanced stars have a velocity dispersion of $\sim 90 \mathrm{~km} \mathrm{~s}^{-1}$, which is approximately constant along the minor axis, while the stars with solar or slightly sub-solar $[\alpha / \mathrm{Fe}]$ abundances have 


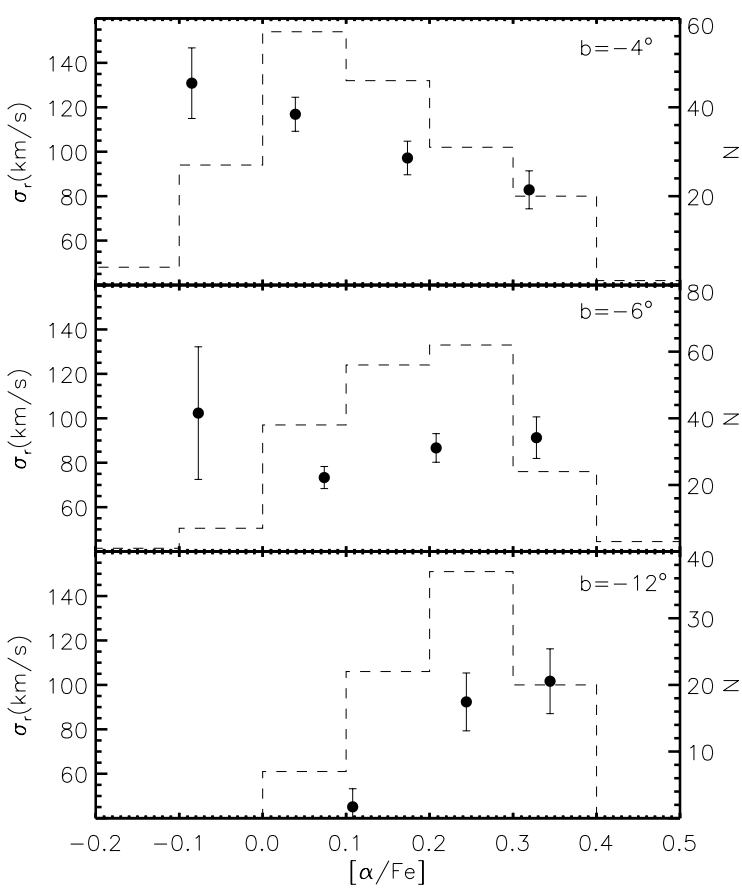

Fig. 17. Dispersion of radial velocity as a function of $[\alpha / \mathrm{Fe}]$, in bins of $0.15 \mathrm{dex}$, for the three fields along the minor axis (filled circles and left axis scale). Overploted as a dashed line is the $[\alpha / \mathrm{Fe}]$ distribution for each field, which scale is given on the right. Globular cluster members in the sample were removed for this particular analysis.

larger velocity dispersion in the inner fields and decreasing velocity dispersion at higher latitude. In the $b=-12^{\circ}$ field the most alpha-poor component disappears altogether. The behaviour of alpha-enhanced stars mimics very closely the (lack of) trend shown by metal-poor component identified as having spheroid kinematics, while alpha-poor stars follow the trend of metal-rich component identified with disk/bar kinematics by Babusiaux et al. (2010). While these trends are interesting, they need to be confirmed with a more complete coverage of the bulge.

\section{Discussion}

We have confirmed the chemical similarity between the alpha element abundances in bulge and thick disk within the errors associated to our homogeneous abundance analysis. An overabundance in alpha elements of $[\alpha / \mathrm{Fe}]=0.30$ dex up to a metallicity of $[\mathrm{Fe} / \mathrm{H}] \sim-0.5$ is observed in both components as an indication of a short timescale of formation, with a chemical enrichment dominated by the contribution of core collapse supernovae. At this metallicity the observed downtrend in the bulge reflects the instance in which the Fe contribution from SNIa becomes important. In the bulge both the trend at low metallicities and the value at which the knee is produced do not vary along the minor axis for latitudes $b<-4$. This can be interpreted as a formation process of the dominating population of the bulge being fairly homogeneous, sharing a similar IMF and formation timescale. Figure 16 shows that these trends are indistinguishable also for two fields at different longitudes $\left(l=1^{\circ}\right.$ and $\left.l=5^{\circ}\right)$ at a fixed latitude of $b=-4^{\circ}$, an indication that the homogeneity is present along the major axis as well.

The chemical similarity of bulge stars and those of the solar neighborhood thick disk, at least for metallicities in which thick and thin disk can be clearly separated, is also remarkably intriguing. Furthermore, Bensby et al. (2010) demonstrated for the first time that inner disk stars show both the dichotomy thin-thick disk and the chemical similarity with the bulge. Either the bulge and thick disk went through similar formation histories or the process which formed the bulge is directly linked to that of the thick disk. Unfortunately the formation history of the thick disk is currently a matter of debate. Therefore, as long as a complete understanding of the formation mechanism of the thick disk is missing, a correct interpretation for the bulge-thick disk connection will be hard to achieve. Particularly interesting, in the context of our results, are the scenarios in which both bulge and thick disk share a fast and early formation such as the scenario discussed in Bournaud et al. (2009) (see also Immeli et al. 2004). They show the properties obtained from simulations in which a thick disk and bulge are formed internally by instabilities of gas-rich clumpy disks in young galaxies. Such clumpy and turbulent rotating disks are in fact observed in galaxies around $z \sim 2$ (Elmegreen et al. 2008). This shared formation mechanism could explain the observed chemical similarities between these components.

The main result our observations provide is that all fields in the bulge follow the same $[\alpha / \mathrm{Fe}]$ vs. $[\mathrm{Fe} / \mathrm{H}]$ relation, and that metal-poor stars correspond to a population enhanced in $\alpha$ elements which shows homogeneous kinematics. These observational facts taken on their own are in accordance with the classical bulge formation through the fast dissipative collapse. This is in contrast with the recent literature claims of pure disk/bar origin of the Galactic bulge (Shen et al. 2010), which would result in shallower $[\alpha / \mathrm{Fe}]$ decline, more similar to that of the thin disk. The most metal-rich part of the population, that shows a downturn in $[\alpha / \mathrm{Fe}]$ has formed on a timescale long enough for SNIa to start contributing significantly with iron peak elements and shows kinematics corresponding to a disk/bar component according to Babusiaux et al. (2010). This component progressively dissapears with increasing distance from the plane.

Altogether, or results provide observational evidence for the coexistance of a classical bulge, within a bar-like component. Dual component bulges have also been observed in external galaxies in the work of Peletier et al. (2007) which shows an old elliptical-like component dominating the Bulge at higher distances from the plane, while a disk-like component is observed in the central regions. In particular, we highlight the recent results from Williams et al. (2011). They show that the bulge of a pure disk galaxy, NGC 3390, rotates perfectly cilindrically, has chemical properties resembling those of the disk and shows no metallicity gradient. On the other hand, the boxy bulge of NGC 1381 shows more complicated rotation properties, is alpha enhanced with respect to the disk and shows a metallicity gradient. These properties for NGC 1381 strikingly resemble those presented here, in a star by star basis, for the Milky Way bulge. In term of simulations, the chemo-dynamical model of Samland \& Gerhard (2003) also predicts a composite bulge: an early collapse component and a metal-rich, alpha-poor population associated to a bar.

Clearly, to settle the issue of the origin of the bulge and to situate it in the context of the formation of the other Galactic components, a detailed analysis of the kinematics and stellar abundances is necessary in a more complete coverage of the bulge and in the inner disk of the Galaxy.

\section{Conclusions}

We have analyzed the abundances of $\mathrm{Mg}, \mathrm{Ca}, \mathrm{Ti}$ and $\mathrm{Si}$ in four fields of the Galactic bulge. We have carried out a homogeneous analysis comparing $[\alpha / \mathrm{Fe}]$ ratios for a large sample of bulge 
stars (650) with those of giants belonging to the thin and thick disk in the Solar vicinity. We also compared bulge $[\alpha / \mathrm{Fe}]$ distributions across the different fields in our sample. Our conclusions can be summarized as follows:

1. We have confirmed the chemical similarity regarding alpha element abundances between the bulge and the thick disk when a homogeneous analysis is done and using only giant stars spectra. Both populations are enhanced compared to the thin disk and show a downtrend in $[\alpha / \mathrm{Fe}]$ starting at a metallicity of $[\mathrm{Fe} / \mathrm{H}] \sim-0.4$. At higher metallicities, close to solar, bulge $[\alpha / \mathrm{Fe}]$ ratios drop to values similar to those of the thin disk.

2. The trends of $[\alpha / \mathrm{Fe}]$ ratios are indistinguishable among our three fields along the bulge minor axis. Analysis on a field at $l=4^{\circ}, b=5^{\circ}$ hints for a homogeneity also present along the major axis. The knee showing the downtrend in $[\alpha / \mathrm{Fe}]$ is observed in all fields at $[\mathrm{Fe} / \mathrm{H}] \sim-0.4$. A more complete mapping of different bulge regions is necessary to confirm the conclusions regarding the major axis lack of gradients as well to explore the $[\alpha / \mathrm{Fe}]$ distribution for inner bulge fields $(b>-4)$.

3. The $[\alpha / \mathrm{Fe}]$ distributions in our different fields show that, while the population of metal-rich stars and low $[\alpha / \mathrm{Fe}]$ ratios observed at $b=-4^{\circ}$ seems to disappear at larger galactic latitudes, trends and distributions at low metallicities $([\mathrm{Fe} / \mathrm{H}]<-0.2)$, as well as velocity dispersion, remain unchanged at different latitudes. Such results are consistent with recent evidences for a dual nature of the bulge.

Our results demand future work to be based on a more complete coverage of the bulge. A better mapping of chemical and kinematical signatures, coupled with the complete morphological characterization to be obtained from a multi-epoch photometric survey such as the VISTA Variables in the Via Lactea (VVV; Minniti et al. 2010), could provide the final step towards the observational characterization of the bulge.

Acknowledgements. We thank the anonymous referee for constructive comments which improved our paper. We acknowledge E. Valenti and S. Lucatello for very useful suggestions and discussions, and M. Williams for fruitful discussions in the topic of boxy-bulges. M.Z., and O.G. acknowledge support by Proyecto Fondecyt Regular 1085278. D.M. acknowledge support by Proyecto Fondecyt Regular 1090213. M.Z. and D.M. are partly supported by the BASAL Center for Astrophysics and Associated Technologies PFB-06, the FONDAP Center for Astrophysics 15010003 and the MIDEPLAN Milky Way Millennium Nucleus P07-021-F. A.A.B. acknowledges financial support by FONDECYT project 3100013. B.B. acknowledges grants from CNPq and FAPESP.

\section{References}

Alonso, A., Arribas, S., \& Martínez-Roger, C. 1999, A\&AS, 140, 261

Alves-Brito, A., Meléndez, J., Asplund, M., Ramírez, I., \& Yong, D. 2010, A\&A, 513, A35

Asplund, M., Grevesse, N., Sauval, A. J., \& Scott, P. 2009, ARA\&A, 47, 481 Athanassoula, E. 2005, MNRAS, 358, 1477

Babusiaux, C., \& Gilmore, G. 2005, MNRAS, 358, 1309

Babusiaux, C., Gómez, A., Hill, V., et al. 2010, A\&A, 519, A77

Bagnulo, S., Jehin, E., Ledoux, C., et al. 2003, The Messenger, 114, 10
Bensby, T., Feltzing, S., Lundström, I., \& Ilyin, I. 2005, A\&A, 433, 185 Bensby, T., Alves-Brito, A., Oey, M. S., Yong, D., \& Meléndez, J. 2010, A\&A, 516, L13

Bournaud, F., Elmegreen, B. G., \& Martig, M. 2009, ApJ, 707, L1

Brown, T. M., Sahu, K., Anderson, J., et al. 2010, ApJ, 725, L19

Bureau, M., Aronica, G., Athanassoula, E., et al. 2006, MNRAS, 370, 753

Carollo, C. M., Scarlata, C., Stiavelli, M., Wyse, R. F. G., \& Mayer, L. 2007, ApJ, 658, 960

Clarkson, W., Sahu, K., Anderson, J., et al. 2008, ApJ, 684, 1110

Daddi, E., Bournaud, F., Walter, F., et al. 2010, ApJ, 713, 686

Debattista, V. P., \& Williams, T. B. 2004, ApJ, 605, 714

Debattista, V. P., Mayer, L., Carollo, C. M., et al. 2006, ApJ, 645, 209

Di Matteo, P., Lehnert, M. D., Qu, Y., \& van Driel, W. 2011, A\&A, 525, L3

Elmegreen, B. G., Bournaud, F., \& Elmegreen, D. M. 2008, ApJ, 688, 67

Förster Schreiber, N. M., Genzel, R., Bouché, N., et al. 2009, ApJ, 706, 1364

Fukugita, M., Hogan, C. J., \& Peebles, P. J. E. 1998, ApJ, 503, 518

Fulbright, J. P., McWilliam, A., \& Rich, R. M. 2006, ApJ, 636, 821

Fulbright, J. P., McWilliam, A., \& Rich, R. M. 2007, ApJ, 661, 1152

Genzel, R., Tacconi, L. J., Eisenhauer, F., et al. 2006, Nature, 442, 786

Genzel, R., Burkert, A., Bouché, N., et al. 2008, ApJ, 687, 59

Gratton, R. G., \& Sneden, C. 1990, A\&A, 234, 366

Gustafsson, B., Edvardsson, B., Eriksson, K., et al. 2008, A\&A, 486, 951

Hopkins, P. F., Bundy, K., Croton, D., et al. 2010, ApJ, 715, 202

Howard, C. D., Rich, R. M., Clarkson, W., et al. 2009, ApJ, 702, L153

Immeli, A., Samland, M., Gerhard, O., \& Westera, P. 2004, A\&A, 413, 547

Kormendy, J. 1993, in Galactic Bulges, ed. H. Dejonghe, \& H. J. Habing, IAU Symp., 153, 209

Kormendy, J., \& Kennicutt, Jr., R. C. 2004, ARA\&A, 42, 603

Lecureur, A., Hill, V., Zoccali, M., et al. 2007, A\&A, 465, 799

McWilliam, A., \& Rich, R. M. 1994, ApJS, 91, 749

McWilliam, A., \& Zoccali, M. 2010, ApJ, 724, 1491

Meléndez, J., Asplund, M., Alves-Brito, A., et al. 2008, A\&A, 484, L21

Minniti, D., \& Zoccali, M. 2008, in IAU Symp., ed. M. Bureau, E. Athanassoula, \& B. Barbuy, 245, 323

Minniti, D., Olszewski, E. W., Liebert, J., et al. 1995, MNRAS, 277, 1293

Minniti, D., Lucas, P. W., Emerson, J. P., et al. 2010, Astron., 15, 433

Nakasato, N., \& Nomoto, K. 2003, ApJ, 588, 842

Nataf, D. M., Udalski, A., Gould, A., Fouqué, P., \& Stanek, K. Z. 2010, ApJ, 721, L28

Pasquini, L., Alonso, J., Avila, G., et al. 2003, in SPIE Conf. Ser. 4841, ed. M. Iye, \& A. F. M. Moorwood, 1682

Patsis, P. A., Skokos, C., \& Athanassoula, E. 2002, MNRAS, 337, 578

Peletier, R. F., Falcón-Barroso, J., Bacon, R., et al. 2007, MNRAS, 379, 445

Peng, Y., Lilly, S. J., Kovač, K., et al. 2010, ApJ, 721, 193

Plez, B. 1998, A\&A, 337, 495

Ramírez, I., \& Meléndez, J. 2005, ApJ, 626, 465

Rangwala, N., \& Williams, T. B. 2009, ApJ, 702, 414

Rattenbury, N. J., Mao, S., Sumi, T., \& Smith, M. C. 2007, MNRAS, 378, 1064

Reddy, B. E., Lambert, D. L., \& Allende Prieto, C. 2006, MNRAS, 367, 1329

Renzini, A. 2009, MNRAS, 398, L58

Rich, R. M., \& Origlia, L. 2005, ApJ, 634, 1293

Rich, R. M., Origlia, L., \& Valenti, E. 2007, ApJ, 665, L119

Ryde, N., Edvardsson, B., Gustafsson, B., et al. 2009, A\&A, 496, 701

Ryde, N., Gustafsson, B., Edvardsson, B., et al. 2010, A\&A, 509, A20

Samland, M., \& Gerhard, O. E. 2003, A\&A, 399, 961

Shen, J., Rich, R. M., Kormendy, J., et al. 2010, ApJ, 720, L72

Sneden, C. 1973, ApJ, 184, 839

Stetson, P. B., \& Pancino, E. 2008, PASP, 120, 1332

Tacconi, L. J., Genzel, R., Neri, R., et al. 2010, Nature, 463, 781

Udalski, A., Paczynski, B., Zebrun, K., et al. 2002, Acta Astron., 52, 1

Weinzirl, T., Jogee, S., Khochfar, S., Burkert, A., \& Kormendy, J. 2009, ApJ, 696, 411

Williams, M. J., Zamojski, M., Bureau, M., et al. 2011, MNRAS, accepted [arXiv: 1102 .2438]

Zoccali, M., Renzini, A., Ortolani, S., et al. 2003, A\&A, 399, 931

Zoccali, M., Lecureur, A., Barbuy, B., et al. 2006, A\&A, 457, L1

Zoccali, M., Hill, V., Lecureur, A., et al. 2008, A\&A, 486, 177 